\title{
Birefringence improvement in azopolymer doped with MFI zeolite nanoparticles
}

\begin{abstract}
Hybrid organic/inorganic materials based on combination of polymers and inorganic nanoparticles (NP) attract considerable attention due to their advantageous electrical, optical, or mechanical properties. Recently it was reported that doping photopolymers with nanoparticles allows to achieve near $100 \%$ net diffraction efficiency in case of conventional holographic recording. Thus, we have synthesized novel organic/inorganic composite materials by incorporating MFI (Mordenite Framework Inverted) type zeolite nanoparticles in an amorphous side-chain azopolymer. A considerable improvement of the photoresponse in thin films of these composite materials has been observed compared to the non-doped samples - nearly $25 \%$ increase of the saturated value of the birefringence. Moreover the photoinduced birefringence is stable in time which allows these materials to be used as media for diffractive optical elements with high efficiency and unique polarization properties.
\end{abstract}

Keywords: Azobenzene polymer; MFI zeolite nanoparticles; Nanocomposite materials; Photoinduced birefringence; Polarimetric measurement

DOI 10.2478/optof-2014-0005

Received June 30, 2014; accepted September 17, 2014

\section{Introduction}

Since the possibility to record high-efficient polarization diffraction gratings in azobenzene materials has been established in 1984 [1], they became subject of intensive re-

\footnotetext{
*Corresponding Author: Lian Nedelchev: Institute of Optical Materials and Technologies, Bulgarian Academy of Sciences, Acad. G. Bonchev Str., bl.109, 1113 Sofia, Bulgaria and College of Telecommunications and Post, 1 Acad. St. Mladenov Str., 1700 Sofia, Bulgaria, E-mail: lian@hctp.acad.bg, Tel.:+359 2 8062214

Dimana Nazarova: Institute of Optical Materials and Technologies, Bulgarian Academy of Sciences, Acad. G. Bonchev Str., bl.109, 1113 Sofia, Bulgaria

Svetlana Mintova: Laboratoire Catalyse \& Spectrochimie, University of Caen, 6, Boulevard du Maréchal Juin, 14050 Caen Cedex, France
}

search. As a result, variety of azobenzene materials and particularly azopolymers have been studied. The recording is based on the selective trans-cis isomerization and the consecutive reorientation of the trans azobenzenes [14]. The photoinduced motion of the azobenzene moieties results in formation of relief gratings on the surface [5-8] and chiral structures in the volume of the azo materials [913], allows to record polarization holographic gratings and optical elements with unique polarization properties [1416] and also to use them for reversible optical storage of information [4, 17-21].

The photoresponse of an azopolymer is mainly characterized by the maximal value of the photoinduced linear birefringence: $\Delta n_{\max }$. As known, the azobenzene materials are optically negative, therefore this parameter is defined as folows: $\Delta n=n_{\perp}-n_{\|}$. The magnitude of $\Delta n_{\max }$ is also closely related to the maximal value of the diffraction efficiency, when recording a polarization hologram in the film. Different methods are used aimed to increase the value of the photoinduced anisotropy - specific architectures of the polymers or thermally assisted recording [22] intended to improve the mobility of the azobenzene molecules. An example of the advantages given by the hybrid organic/inorganic materials has been recently reported: photopolymers doped with nanoparticles (NP) make it possible to achieve near $100 \%$ net diffraction efficiency in case of conventional holographic recording $[23,24]$. Similar effect is observed also by Leite et al. in nanocomposites both for holographic and sensor applications [25].

For this reason, we have investigated organic/inorganic composite materials prepared by incorporating pure silica Mordenite Framework Inverted (MFI) zeolite nanoparticles in an amorphous azopolymer. The trans-cis isomerization of azobenzene chromophores requires a free volume and if it is limited, the reorientation of the azobenzenes is impeded resulting in lower $\Delta n_{\max }$ [26]. When inorganic nanoparticles are introduced in the polymer film, the interlacing of the azobenzene molecules close to the surface of the NP is reduced which results in enhanced mobility.

Here we present a study of the photoresponse of the composite films i.e. the value of $\Delta n_{\max }$ depending on the concentration of the doped MFI nanoparticles. 


\section{Experimental procedures}

\subsection{Synthesis and characterization of the MFI zeolite nanoparticles}

Pure silica MFI-type zeolite was synthesized through a procedure described in [27-29]. After completing the synthesis, the crystalline suspension was purified by highspeed centrifugation in three subsequent cycles and redispersed in water. The size of the nanoparticles was determined by Transmission Electron Microscopy (TEM) using a JEOL 2010 FEG operating at $200 \mathrm{kV}$. As seen in Fig. 1, the nanocrystals are rectangular with sizes about $15 \times 25 \mathrm{~nm}$ and exhibit almost plate-like morphology.

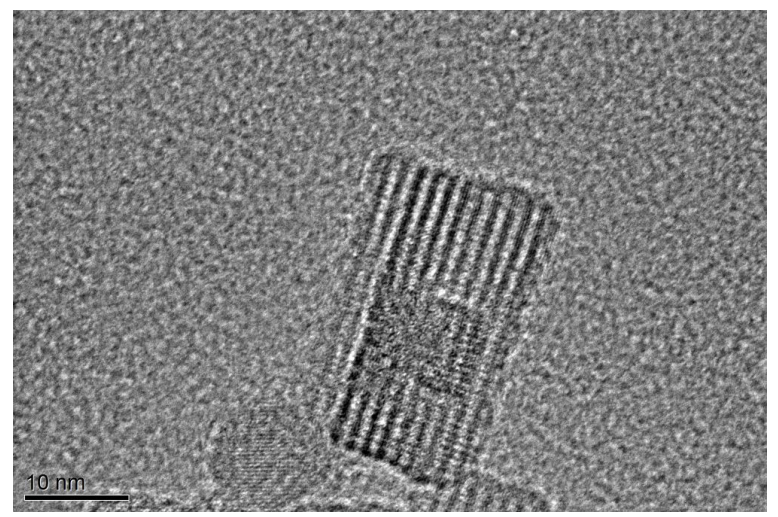

Figure 1: TEM image of a MFI zeolite nanoparticle.

\subsection{Chemical structure of the azopolymer matrix and preparation of the thin films}

The azopolymer used as organic host in our nanocomposite samples is an amorphous side-chain polymer and its structure is shown in Fig. 2. The polymer is denoted as $\mathrm{P}_{1}$ and was synthesized according to a procedure described earlier [30]. Three stages can be outlined in this process: (a) synthesis of an azodye; (b) preparation of a chromophore monomers from the azodye and acryloyl chloride; (c) polymerization of the chromophore monomers. During the first stage the azodye 4-(4-hydroxyphenylazo)benzonitrile was prepared by dissociation of 4aminobenzonitrile and coupling with phenol using standard technology. Then by etherification of the dye with acryloyl chloride the azodye monomer was obtained. Finally, the polymer was synthesized by radical-type polymerization. The molecular weight of $\mathrm{P}_{1}$ as determined by
GPC is $M_{w}=14600$ and its glass transition temperature measured by DSC is $T_{g}=106^{\circ} \mathrm{C}$.

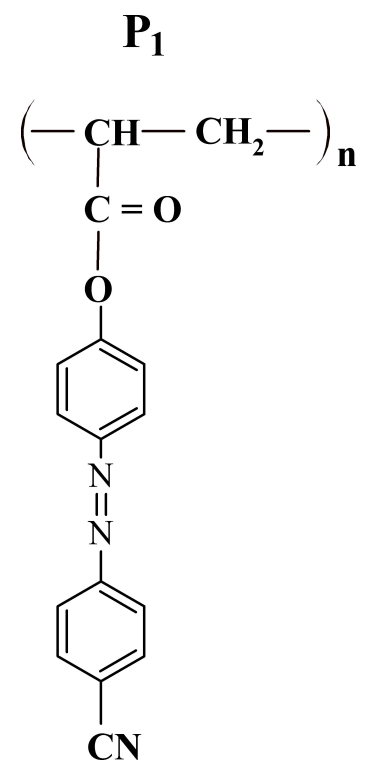

Figure 2: Structure of the azopolymer $\mathrm{P}_{1}$.

In order to prepare thin films for optical testing, each polymer was dissolved in 1,2-dichloroethane, MFI zeolite nanoparticles were added to obtain the desired concentrations and the solutions were spin coated at $1500 \mathrm{rpm}$ onto glass substrates. Apart from the films made from nondoped (or "pure") polymers, five other NP concentrations relative to the azopolymer were used $-C=0.5,1,2,5$ and 10 wt. \%. All of the obtained samples are with good optical quality i.e. no scattering or surface defects are visually observed.

\subsection{Determination of the samples thickness}

The precise value of the samples thickness, denoted as $d$, is essential to accurately determine their optical properties, namely the absorbance spectra and the birefringence. The thickness is measured by high-precision Talystep profilometer with $1 \mathrm{~nm}$ vertical and $0.1 \mu \mathrm{m}$ horizontal resolution, additionally upgraded with a digital recording device connected to a PC. A groove is made on the surface of each sample, inside which the nanocomposite film is removed and the distance between the "plateau" and the bottom of the groove corresponds to the layer's thickness. In order to ensure that the glass substrate is not affected, we use a tip with hardness lower than the hardness of glass. On the other hand, the obtained profiles clearly show that the 
polymer film is completely removed at the bottom of the groove.

In spite of the same conditions during the spin coating of all samples (same amounts of polymer/solvent and also same rotation speed), there are still some variations in the thicknesses of the films and they are in the range $0.5-0.7 \mu \mathrm{m}$, as seen in Table 1. The uncertainty of the thickness measurement for our samples, quantified by its dispersion, is $5 \%$.

\subsection{Spectrophotometric characterization}

The samples absorbance spectra were determined using a Varian Cary 5E spectrophotometer. The data directly obtained from the measurement were the spectra of transmittance $T(\lambda)$ which were then transformed to absorbance $\alpha$ using the Beer-Lambert law i.e.

$$
\alpha=-\frac{1}{d} \log (T) .
$$

As indicated by Eq. 1, to make a correct comparison of the optical properties of the nanocomposite films, the data about the film thickness $d$ are very important.

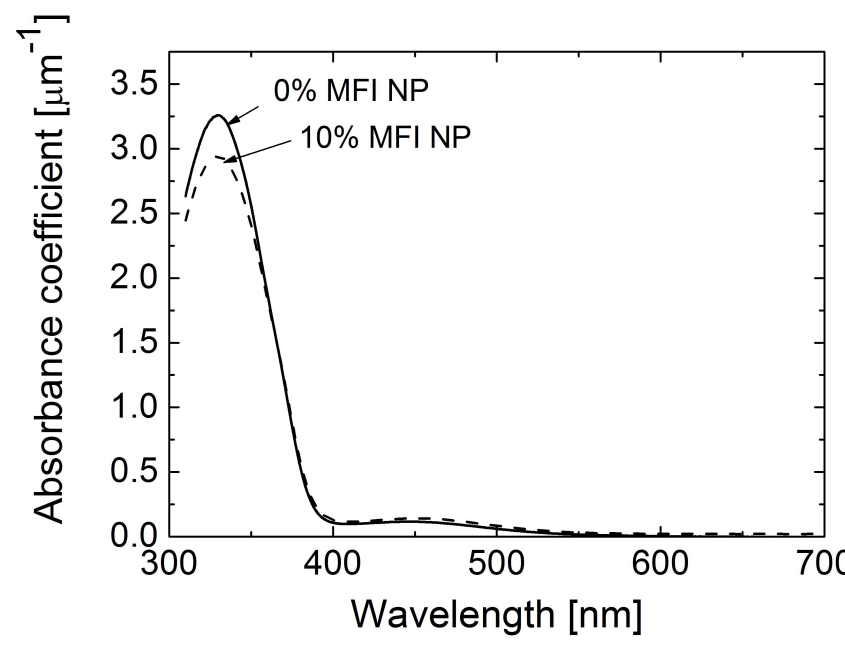

Figure 3: Absorbance spectra of the non-doped azopolymer sample (-) and the sample with highest concentration (10 wt.\%) of the MFI NP (- - -).

Fig. 3 presents the spectra of absorbance for the samples with $0 \%$ and 10 wt.\% concentration of the doped nanoparticles. The spectra indicate that the addition of MFI NP does not shift the peak of absorbance and its maximum is at $330 \mathrm{~nm}$. It is also seen that doping even $10 \%$ of NPs does not give raise to scattering outside the absorbance band (beyond $500 \mathrm{~nm}$ ) i.e. there is no increase of the absorbance coefficient. This is also confirmed by the visual observation of the films - they are with good optical quality and high transparency.

\section{Results}

\subsection{Polarimetric setup and calculation}

The photoresponse was evaluated by classical polarimetric setup where the sample is positioned between two crossed polarizers with axes oriented at $\pm 45^{\circ}$ with respect to the vertical. Vertically polarized beam from a DiodePumped Solid State (DPSS) laser at $473 \mathrm{~nm}$ and intensity $400 \mathrm{~mW} / \mathrm{cm}^{2}$ was used to induce birefringence in the film. It was probed with a He-Ne laser at $633 \mathrm{~nm}$ and intensity $20 \mathrm{~mW} / \mathrm{cm}^{2}$, which does not practically influence the recording, as $633 \mathrm{~nm}$ is outside the absorbance band of the samples (see Fig. 3). From the intensity $I$ of the probe beam transmitted through the system, the value of the photoinduced birefringence can be easily determined using the following equation:

$$
\Delta n=\frac{\lambda_{\text {probe }}}{\pi d} \cdot \arcsin \sqrt{I / I_{0}},
$$

where $d$ is the thickness of the layer and $I_{0}$ is the intensity of the probe beam before the start of the recording and at parallel polarizers. The intensity is measured with digital optical power meter (Thorlabs PM100) and recorded on a computer in real time.

\subsection{Measurement of the photoinduced birefringence}

The experimental results obtained with this experimental setup are presented in Fig. 4a. For each sample the measurement cycle is $600 \mathrm{~s}$ (or $10 \mathrm{~min}$ ) divided into three periods: (i) initial stage of $60 \mathrm{~s}$ before the recording is started to evaluate the background noise, (ii) $300 \mathrm{~s}$ of irradiation with the pump laser, sufficient to reach saturation of the photoinduced birefringence, and (iii) $240 \mathrm{~s}$ of relaxation, after the recording beam has been stopped, to estimate the stability of the recorded birefringence.

It is easier to compare the data for all samples if we define a parameter called "Increase ratio" $(I R)$ in the following way:

$$
I R(C)=\frac{\Delta n_{\max }(C)-\Delta n_{\max }(0)}{\Delta n_{\max }(0)} \cdot 100 \%,
$$

where $\Delta n_{\max }(C)$ is the maximal saturated value of the photoinduced birefringence for nanocomposite film with con- 
Table 1: Data for the thickness of all investigated samples.

\begin{tabular}{lllllll}
\hline $\begin{array}{l}\text { Concentration of MFI zeolite NP } \\
\text { relative to the azopolymer, } C \text { [\%] }\end{array}$ & 0 & 0.5 & 1 & 2 & 5 & 10 \\
\hline Sample thickness, $d[\mu \mathrm{m}]$ & 0.57 & 0.47 & 0.63 & 0.47 & 0.51 & 0.68 \\
\hline
\end{tabular}

centration of the NP relative to the azopolymer $C$, and $\Delta n_{\max }(0)$ is the maximal saturated value of the photoinduced birefringence for the non-doped (or "pure") film. For our samples $\Delta n_{\max }(0)=0.021, \Delta n_{\max }(1 \%)=0.026$ and $\Delta n_{\max }(10 \%)=0.022$. As clearly seen in Fig. $4 \mathrm{~b}$, the values of $\Delta n_{\max }$ for all the nanocomposite films exceed the corresponding value for the pure polymer film (i.e. $I R>0$ ). The increase of the birefringence is highest $(I R \approx 25 \%)$ for the sample with concentration of the MFI nanoparticles relative to the azopolymer $\mathrm{P}_{1} C=1 \%$. The peak in the dependence $I R(C)$ is most likely related to the increase of the mobility of azochromophores caused by the free volume created at the contact surface between the azo molecules and the nanoparticles. At higher concentrations of the dopant though ( 5 and $10 \mathrm{wt}$. \%), the value of $\Delta n_{\max }$ starts to decrease. A possible reason for this effect is the fact that the NP themselves are not photoactive and do not directly contribute to the build-up of the birefringence. Evidently, at increased concentrations of the NPs this effect prevails.

Other parameters that describe the response of a photobirefringent material are the response time and the time stability. Response time $\tau$ is a parameter defined as the time needed to reach $80 \%$ of the saturated birefringence value $\Delta n_{\max }$ at a given laser intensity [19]. Hence, the response time is a convenient single parameter that allows to compare the time and energy required to record certain value of birefringence in different layers. The time stability is calculated as the percent ratio of the birefringence retained by the sample after certain time of relaxation (in this case $240 \mathrm{~s}$ ) to the maximal birefringence value $\Delta n_{\max }$. Our experimental data indicate that the response time is in the range $18-23 \mathrm{~s}$, the time stability is about $86-88 \%$ and the two parameters are virtually independent on the NP concentration $C$.

\section{Discussion}

The present observation of the enhancement of the photoresponse on doping MFI nanoparticles in an amorphous azopolymer confirms our earlier studies where azopolymers were doped with $\mathrm{ZnO}[31,32]$ and $\mathrm{SiO}_{2}$ nanoparticles [33]. It is also supported by the results published by
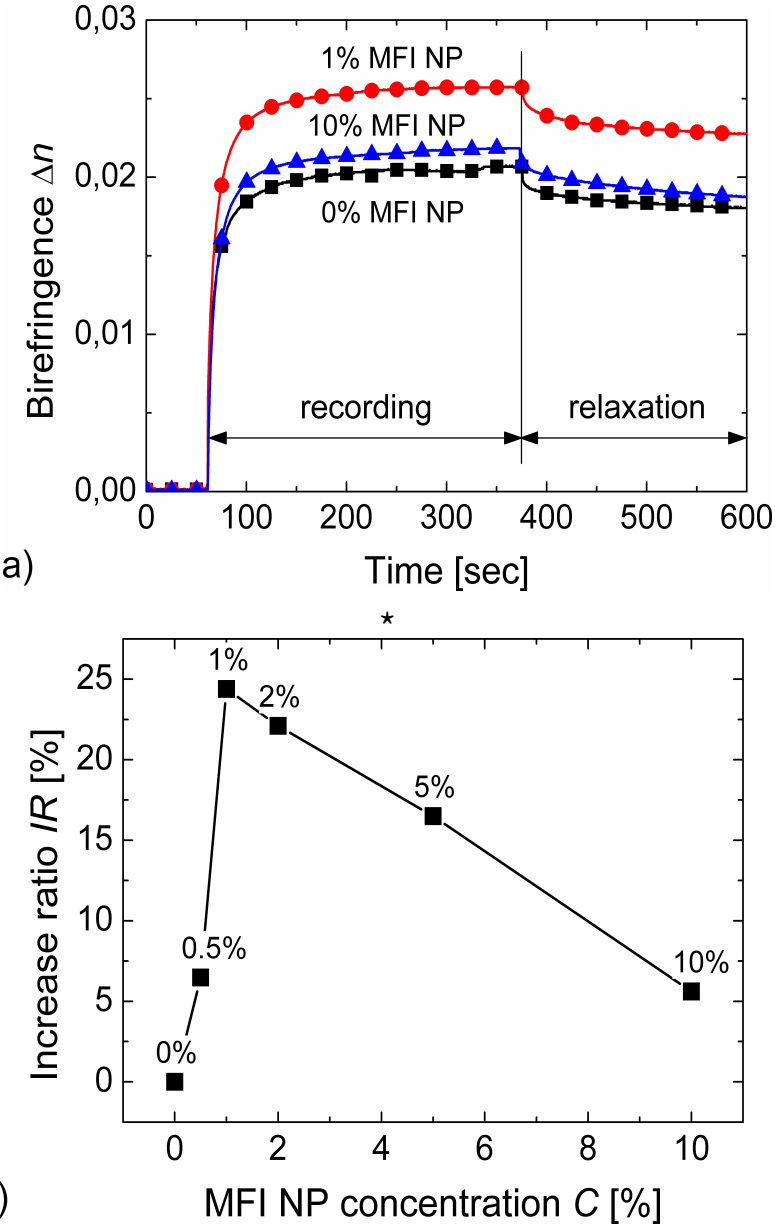

Figure 4: a) Time dependence of the photoinduced birefringence in non-doped and doped samples ( $1 \%$ and $10 \%$ concentration of the MFI nanoparticles) based on the amorphous azopolymer $\mathrm{P}_{1}$ $\left.\left(I_{\text {recording }}=400 \mathrm{~mW} / \mathrm{cm}^{2}, \lambda=473 \mathrm{~nm}\right) ; \mathrm{b}\right)$ Increase ratio $I R$ vs. the concentration of MFI nanoparticles $C$ (the filled squares are experimental data points, the lines are given to guide the eye).

Zhou et al. [34] in polymer containing azo group and MMA doped with silver NPs - it was found that at certain wavelengths, specifically $442 \mathrm{~nm}$, there is a considerable increase in the value of the photo-induced transmittance.

Another interesting point is that MFI zeolite nanoparticles could be used in medicine, in particular in toxicology [35]. It is possible that the nanocomposites of the photo addressable azopolymer and these nanoparticles can find applications in photoselective disease treatment. 
To summarize, we observed a considerable improvement of the photoresponse in composite materials consisting of azopolymer doped with MFI zeolite nanoparticles in relation to the non-doped samples - approximately $25 \%$ increase of the saturated value of the birefringence. These anisotropic nanocomposites can be used as media for polarization holographic storage or recording of diffractive optical elements with high efficiency and unique polarization properties.

Acknowledgement: The authors gratefully acknowledge the support of COST Action MP1205 "Advances in Optofluidics: Integration of Optical Control and Photonics with Microfluidics”. The authors are indebted to Ms Anna Lalova for the measurement of the absorbance spectra of the samples.

\section{References}

[1] T. Todorov, L. Nikolova and N. Tomova, Polarization Holography 1. A new high-efficiency organic material with reversible photoinduced birefringence, Appl. Opt. 23, 1984, 4309.

[2] Nikolova L., Ramanujam P. S., Polarization Holography, Cambridge University Press, Cambridge, 2009

[3] M. Eich and J. H. Wendorff, Erasable holograms in polymeric liquid-crystals, Makromol. Chem. - Rapid Comm. 8, 1987, 467.

[4] A. Natansohn, P. Rochon, J.Gosselin and S.Xie, Azo polymers for reversible optical storage. 1. Poly[4'-[[2(acryloyloxy)ethyl]ethylamino]-4-nitroazobenzene], Macromolecules 25, 1992, 2268.

[5] D. Y. Kim, L. Li, X. L. Jiang, V. Shivshankar, J. Kumar and S. K. Tripathy, Polarized laser induced holographic surface relief gratings on polymer films, Macromolecules 28, 1995, 8835.

[6] N. K. Viswanathan, D. Y. Kim, S. Bian, J. Williams, W. Liu, L. Li, L. Samuelson and S. K. Tripathy, Surface relief structures on azo polymer films, J. Mat. Chem. 9, 1999, 1941.

[7] T. Fukuda, K. Sumaru, T. Kimura and H. Matsuda, Photofabrication of surface relief structure - mechanism and application, J. Photoch. Photobio. A 145, 2001, 35.

[8] J.Zhou, J. Yang, Y. Ke, J. Shen, Q. Zhang and K. Wang, Fabrication of polarization grating and surface relief grating in crosslinked and non-crosslinking azopolymer by polarization holography method, Opt. Mat. 30, 2008, 1787.

[9] L. Nikolova, L. Nedelchev, T. Todorov, Tz. Petrova, N. Tomova, V. Dragostinova, P. S. Ramanujam and S. Hvilsted, Self-induced light polarization rotation in azobenzene-containing polymers, Appl. Phys. Lett. 77, 2000, 657.

[10] G. Iftime, F. L. Labarthet, A. Natansohn and P. Rochon, Control of chirality of an azobenzene liquid crystalline polymer with circularly polarized light, J. Amer. Chem. Soc. 122, 2000, 12646.

[11] K. G. Yager and C. J. Barrett, Novel photo-switching using azobenzene functional materials, J. Photochem. Photobiol. A Chem. 182, 2006, 250.

[12] Z. Zheng, L. Wang, Z. Su, J. Xu, J. Yang and Q. Zhang, Photoinduced chirality in achiral liquid crystalline polymethacrylates containing bisazobenzene and azobenzene chromophores, J. Photoch. Photobio. A 185, 2007, 338.

[13] J. del Barrio, R. M. Tejedor and L. Oriol, Thermal and light control of the chiral order of azopolymers, Eur. Polym. J. 48, 2012, 384.

[14] L. Nikolova, T. Todorov, M. Ivanov, F. Andruzzi, S. Hvilsted and P. S. Ramanujam, Polarization holographic gratings in side-chain azobenzene polyesters with linear and circular photoanisotropy, Appl. Opt. 35, 1996, 3835.

[15] L. Nedelchev, T. Todorov, L. Nikolova, Tz. Petrova, N. Tomova and V. Dragostinova, Characteristics of high-efficient polarization holographic gratings, Proc. SPIE 4397, 2001, 338.

[16] P. S. Ramanujam, C. Dam-Hansen, R. H. Berg, S. Hvilsted and L. Nikolova, Polarisation-sensitive optical elements in azobenzene polyesters and peptides, Opt. Las. Eng. 44, 2006, 912.

[17] S. Hvilsted, F. Andruzzi, C. Kulinna, H. W. Siesler and P. S. Ramanujam, Novel side-chain liquid-crystalline polyester architecture for reversible optical storage, Macromolecules 28, 1995, 2172.

[18] P. H. Rasmussen, P. S. Ramanujam, S. Hvilsted and R. H. Berg, A remarkably efficient azobenzene peptide for holographic information storage, J. Amer. Chem. Soc. 121, 1999, 4738.

[19] L. Nedelchev, A. S. Matharu, S. Hvilsted and P. S. Ramanujam, Photoinduced anisotropy in a family of amorphous azobenzene polyesters for optical storage, Appl. Opt. 42, 2003, 5918.

[20] N. C. R. Holme, S. Hvilsted, E. Lőrincz, A. Matharu, L. Nedelchev, L. Nikolova and P. S. Ramanujam, Azobenzene Polyesters for Polarization Holographic Storage: Part I Materials and Characterization, in: H. Nalwa (Ed.), Handbook of Organic Electronics and Photonics, Amer. Sci. Publ., Valencia CA, 2008, pp. 184-211.

[21] H. Audorff, K. Kreger, R. Walker, D. Haarer, L. Kador and H. W. Schmidt, Holographic Gratings and Data Storage in Azobenzene-Containing Block Copolymers and Molecular Glasses, Adv. Polym. Sci. 228, 2010, 59.

[22] M. Ivanov, D. Ilieva, G. Minchev, Ts. Petrova, V. Dragostinova, T. Todorov and L. Nikolova, Temperature-dependent light intensity controlled optical switching in azobenzene polymers, Appl. Phys. Lett. 86, 2005, 181902.

[23] N. Suzuki and Y. Tomita, Silica-nanoparticle-dispersed methacrylate photopolymers with net diffraction efficiency near 100\%, Appl. Opt. 43, 2004, 2125.

[24] Y. Tomita, N. Suzuki and K. Chikama, Holographic manipulation of nanoparticle distribution morphology in nanoparticledispersed photopolymers, Opt. Lett. 30, 2005, 839.

[25] E. Leite, I. Naydenova, S. Mintova, L. Leclercq and V. Toal, Photopolymerizable nanocomposites for holographic recording and sensor application, Appl. Opt. 49, 2010, 3652.

[26] S. M. Shah, C. Martini, J. Ackermann and F. Fages, Photoswitching in azobenzene self-assembled monolayers capped on zinc oxide: Nanodots vs nanorods, J. Colloid Interface Sci. 367, 2012, 109.

[27] S. Mintova, N. H. Olson, J. Senker and T. Bein, Mechanism of the Transformation of Silica Precursor Solutions into Si-MFI Zeolite, Angew. Chem. 41, 2002, 2558.

[28] T. Babeva, R. Todorov, S. Mintova, T. Yovcheva, I. Naydenova and V. Toal, Optical properties of silica MFI doped acrylamidebased photopolymer, J. Opt. A: Pure Appl. Opt. 11, 2009, 024015.

[29] T. Babeva, H. Awala, M. Vasileva, J. El Fallah, K. Lazarova and S. Mintova, Pure silica MFI zeolite films as antireflection coatings, Bulg. Chem. Commun. 45B, 2013, 18. 
[30] G. Martinez-Ponce, Ts. Petrova, N. Tomova, V. Dragostinova, T. Todorov and L. Nikolova, Investigations on photoinduced processes in a series of azobenzene-containing side-chain polymers, J. Opt. A: Pure Appl. Opt. 6, 2004, 324.

[31] L. Nedelchev, D. Nazarova, V. Dragostinova and D. Karashanova, Increase of photoinduced birefringence in a new type of anisotropic nanocomposite: azopolymer doped with $\mathrm{ZnO}$ nanoparticles, Opt. Lett. 37, 2012, 2676.

[32] L. Nedelchev, D. Nazarova and V. Dragostinova, Photosensitive organic/inorganic azopolymer based nanocomposite materials with enhanced photoinduced birefringence, J. Photochem. Photobiol. A: Chem. 261, 2013, 26.
[33] D. Nazarova, L. Nedelchev, P. Sharlandjiev and V. Dragostinova, Anisotropic hybrid organic/inorganic (azopolymer/ $/ \mathrm{SiO}_{2} \mathrm{NPs}$ ) materials with enhanced photoinduced birefringence, Appl. Opt. 52, 2013, E28.

[34] J. Zhou, J. Yang, Y. Sun, D. Zhang, J. Shen, Q. Zhang, K. Wang, Effect of silver nanoparticles on photo-induced reorientation of azo groups in polymer films, Thin Solid Films 515, 2007, 7242.

[35] K. Bhattacharya, P. C. Naha, I. Naydenova, S. Mintova and H. J. Byrne, Reactive oxygen species mediated DNA damage in human lung alveolar epithelial (A549) cells from exposure to noncytotoxic MFI-type zeolite nanoparticles, Toxicology Letters 215, 2012, 151. 